\title{
The Feasibility of Reducing and Measuring Sedentary Time among Overweight, Non-Exercising Office Workers
}

\author{
Sarah Kozey-Keadle, ${ }^{1}$ Amanda Libertine, ${ }^{1}$ John Staudenmayer, ${ }^{2}$ and Patty Freedson ${ }^{1}$ \\ ${ }^{1}$ Department of Kinesiology, University of Massachusetts, Amherst, MA 01003, USA \\ ${ }^{2}$ Department of Mathematics and Statistics, University of Massachusetts, Amherst, MA 01003, USA
}

Correspondence should be addressed to Patty Freedson, psf@kin.umass.edu

Received 11 August 2011; Revised 20 September 2011; Accepted 22 September 2011

Academic Editor: Dale Bond

Copyright (C) 2012 Sarah Kozey-Keadle et al. This is an open access article distributed under the Creative Commons Attribution License, which permits unrestricted use, distribution, and reproduction in any medium, provided the original work is properly cited.

\begin{abstract}
This study examined the feasibility of reducing free-living sedentary time (ST) and the convergent validity of various tools to measure ST. Twenty overweight/obese participants wore the activPAL (AP) (criterion measure) and ActiGraph (AG; 100 and 150 count/minute cut-points) for a 7-day baseline period. Next, they received a simple intervention targeting free-living ST reductions (7-day intervention period). ST was measured using two questionnaires following each period. ST significantly decreased from $67 \%$ of wear time (baseline period) to $62.7 \%$ of wear time (intervention period) according to AP $(n=14, P<0.01)$. No other measurement tool detected a reduction in ST. The AG measures were more accurate (lower bias) and more precise (smaller confidence intervals) than the questionnaires. Participants reduced ST by $\sim 5 \%$, which is equivalent to a 48 min reduction over a 16-hour waking day. These data describe ST measurement properties from wearable monitors and self-report tools to inform sample-size estimates for future ST interventions.
\end{abstract}

\section{Introduction}

Sedentary behavior is defined as energy expenditure between 1-1.5 metabolic equivalents (METs) while sitting or reclining [1] and accounts for the majority of occupational, transportation, and discretionary time [2]. Using data from the National Health and Nutrition Examination Survey, Matthews et al. reported that $54 \%$ of waking hours were sedentary [3]. Among healthy, predominantly overweight individuals, $62 \%-68 \%$ of waking hours are spent in sedentary behaviors [4]. A growing body of evidence shows that sedentary time is associated with an increased risk of obesity, chronic disease, and mortality [1]. However, to date, the majority of evidence linking sedentary behavior to adverse health consequences is cross-sectional [5-7], from which causality cannot be determined. However, a recent review by Thorp et al. [8] concluded that there is some prospective evidence that supports relationships between sedentary behavior, mortality, and health outcomes. Studies have shown that sedentary time is associated with increased risk for type II diabetes [9-11] and mortality [12]. Other studies report no association or suggest reverse causality between sedentary time, obesity, and insulin resistance [13, 14]. Studies often failed to adjust for physical activity and BMI, which may explain the disparate results [8]. In addition, the majority of these studies used surrogate measures of sedentary time (e.g., TV viewing) and self-report measures, which may not accurately measure sedentary time.

Sedentary time is frequently estimated from surrogate measurements such as time spent watching television (TV) $[10,12,15]$. Robust positive relationships have been reported between TV viewing and poor health outcomes including risk of diabetes and premature mortality [16]. However, TV viewing is also associated with increased energy intake, and markers of poor health that may confound the association between sedentary time and metabolic health $[17,18]$. Furthermore, while TV viewing is correlated with sedentary time among unemployed individuals, it is not for those who are employed suggesting that TV viewing may be a poor surrogate measure for overall sedentary time [19]. Selfreport questionnaires, including those that measure domainspecific [20] and single-item [21] sitting time, are also 
available. A recent review by Healy and colleagues suggests that existing questionnaires may be acceptable for establishing cross-sectional associations but may not be acceptable for prospective or intervention trials [22]. The authors note a paucity of data on the absolute agreement of sedentary time estimates from self-report questionnaires, and few studies have compared sedentary time questionnaires to a valid criterion measure [22].

Activity monitors are attractive tools to measure sedentary time. To date, though, few studies (i.e., 3 of 48 included in the Thorp review) have used activity monitors for sedentary behavior research [8]. The ActiGraph (AG), using the cut-point of 100 counts. $\mathrm{min}^{-1}$ (AG100), is the most commonly used objective tool to assess sedentary time. Previous research from our laboratory showed that the activPAL (AP) activity monitor is more accurate, precise, and sensitive to detecting changes in sedentary time than AG using a number of sedentary time cut-points ranging from 50 to 250 counts $\cdot \mathrm{min}^{-1}$ [23]. However, our validation results were based on two, 6-hour direct observation sessions, and do not include factors such as day-to-day variability that is important to quantify for intervention studies designed to decrease sedentary time. To date, no studies have used the AP, a criterion measure of sedentary time [24], to compare the validity of existing measurement tools over a 7-day period or to assess the ability of existing measurement tools to detect changes in free-living sedentary time.

While an abundance of evidence suggests that sedentary time is associated with poor health outcomes, we do not know the feasibility of reducing sedentary time, the validity of existing measurement tools over a 7-day period, or the ability of existing measurement tools to detect changes in free-living sedentary time $[22,25]$. This study addressed these knowledge gaps with the following three aims. First, we determined if a simple one-week sitting time intervention decreased sedentary time as measured by the criterion AP among nonexercising, overweight/obese individuals with sedentary occupations. We compared sedentary time preand postintervention for the total week and for weekend and weekdays. Second, we compared whether or not existing questionnaires and activity monitors detected reductions in sedentary time following the 7-day intervention. Third, we compared the convergent validity of the AP, the AG, and the questionnaires.

\section{Methods}

2.1. Participants. Participants were recruited from the University of Massachusetts, Amherst, and local communities. Eligible participants were between 20 and 60 years of age, overweight or obese with a body mass index (BMI) between 25 and $45 \mathrm{~kg} \cdot \mathrm{m}^{-2}$, inactive (i.e., participating in moderateto-vigorous physical activity [MVPA] $<3$-days per week for $<20$ minutes per session in the preceding six months), and employed in jobs where the majority of their day was sedentary (i.e., participants self-reported over $75 \%$ of their work day as sedentary). Potential participants completed a telephone screening to determine eligibility.

\subsection{Study Protocol}

2.2.1. Visit 1. Eligible participants reported to the laboratory at the University of Massachusetts for an informed consent visit. Participants read and signed an informed consent document (ICD) that was approved by the Institutional Review Board at the University of Massachusetts. After signing the ICD, height and weight were measured. The average (SD) age was $46.5(10.8)$ years and BMI was $33.7(5.6) \mathrm{kg} \cdot \mathrm{m}^{-2}$. Seventy-five percent $(15 / 20)$ of the participants were female. Participants were shown the activity monitors (described below) and were provided with detailed verbal and written instructions on proper monitor placement. They were instructed to wear the monitors for a 7-day period while maintaining habitual activity levels. This was the baseline period.

2.2.2. Visit 2. After wearing the monitors for 7 days, participants returned to the laboratory, and the activity monitor data were downloaded. Participants completed two selfreport questionnaires (described below) with questions about sitting time during the previous 7 days (baseline period).

2.2.3. Intervention. A researcher provided the participant with information about the potential health risks associated with sedentary time and the benefits associated with increasing light-intensity activity. Participants were given a packet that contained a list of strategies to reduce sedentary time and a checklist to monitor sedentary time for each of the next 7 days. The document outlining strategies to reduce sedentary time included an extensive list of ways to replace sedentary time with light-intensity activity. They are the following:

\section{At Home}

(i) Walk while talking on the phone.

(ii) Walk your dog an extra 10 minutes each day.

(iii) Do dishes by hand instead of using the dishwasher.

(iv) Stand during commercials (remain standing an extra minute after).

(v) Do a little extra housework.

(vi) When grocery shopping, walk up and down each isle, even doing it twice to walk longer and to pick up grocery items you may have forgotten the first time.

(vii) Walk up and down stairs a couple times a day.

(viii) When you are carrying things in from the car (e.g., groceries) take more frequent trips with only one bag at a time.

(ix) Walk to get the mail, instead of driving by shovel instead of using a snow blower.

(x) Mow your lawn (even better get a nonmotorized mower).

(xi) Wash your car (no drive through!). 
At Work

(i) Stand to answer telephone.

(ii) Take a 5-minute walk/stand break each hour.

(iii) Hand-deliver a message to a coworker instead of emailing.

(iv) Take the stairs (start with walking 2 floors then taking elevator if your building is tall).

(v) Use restroom on a different floor.

(vi) Eat your lunch outside or somewhere other than your desk.

\section{Recreation and Transportation}

(i) Choose active recreation instead of going to a movie (e.g., bowling, pool, and darts).

(ii) Volunteer to plant trees or start a garden at home.

(iii) Volunteer to walk a dog, play with kids in need, or help habitat for humanity.

(iv) Take the bus or other public transportation when possible.

(v) Go for a hike or a picnic instead of going for a scenic drive.

The packet also included a form asking participants about specific barriers in their free-living environment that would inhibit reductions in sedentary time. They were then counseled on specific ways to overcome those barriers. In addition, they were given a daily checklist reminding them to break up sedentary time by reporting if they stood or walked for five or more minutes for each hour of the day. Finally, participants were provided a pedometer to wear for the next 7 days and given a goal of attaining 7500 steps/day, the lower boundary for "somewhat active" behavior [26]. Participants were instructed to accumulate the steps in 5-15 minute bouts over the course of the day rather than one large bout of activity. Participants were instructed to wear the AG and AP activity monitors for another 7-day period and were asked to follow the recommendations to reduce sedentary time. This was the intervention period.

2.2.4. Visit 3. Participants returned the monitors on the third visit and completed the same two self-report questionnaires completed at visit one. These questionnaires asked about time spent sedentary in the previous 7 days (intervention period). After completing the questionnaires, participants were asked the following questions about the intervention period: "Was the pedometer step per day goal helpful in meeting your goals? Why or why not?", "Was the daily checklist helpful for meeting your goals?", and "Did you fill out the checklist (circle the one that best applies)" (1) once a day (2) as you completed activity (3) every couple of days (4) once in the week.

\subsection{Measurements}

2.3.1. ActivPAL Activity Monitor (PAL Technologies, Glasgow, Scotland, UK). This is a small $(2.0 \times 1.4 \times 0.3$ inches $)$ and light (20.1 grams) uniaxial accelerometer-based device that was worn anteriorly on right mid-thigh and held in place by nonallergenic adhesive tape. This device uses accelerometerderived information about thigh position to estimate time spent in different body positions (i.e., sitting/lying, standing and stepping). Data were collected for a one-week period and processed in 15-second epochs using activPAL software (version 5.8.3). We previously validated the activPAL monitor for measuring free-living sedentary time in the same subjects as the current study [23]. The activPAL was valid and precise with a bias of $2.8 \%$ and an $R^{2}$ value of 0.94 compared to direct observation [23]. The monitor was also sensitive to reductions in sedentary time [23]. In a laboratory-based validation Grant et al. [24] reported a mean percentage difference between sedentary time from the monitor and direct observation of $0.19 \%$ and the mean difference for total time spent upright was $-0.27 \%$.

\subsubsection{ActiGraph GT3X Activity Monitor (Actigraph LLC,} Pensacola, Fla, USA). This is a small $(1.5 \times 1.44 \times 0.7$ inches) and light (28 grams) accelerometer that was worn on the right hip and secured by an elastic belt. The monitor was initialized using ActiLife software version 4.2 and firmware version 2.1.0. The monitor was initialized to record vertical accelerations in 1-second epochs with the lowfrequency extension option activated. Count cut-points of 100 counts $\cdot \mathrm{min}^{-1}$ (AG100) and 150 counts $\cdot \mathrm{min}^{-1}$ (AG150) were used to define sedentary time. The Freedson cutpoint of 1952 counts $\cdot \mathrm{min}^{-1}$ was used to define moderate to vigorous physical activity (MVPA) [27].

\subsubsection{Omron Pedometer HJ720-ITC (Omron Healthcare,} Bannockburn, Ill, USA). Pedometers have been used to provide referent goals for individuals to estimate activity levels. For example, $<5000$ steps/day is sedentary, 5000-7499 steps/day is low active, and $>10,000$ steps/day is active [26]. The pedometer provided a self-monitoring tool to facilitate compliance with sedentary reduction recommendations, but since pedometer steps are not a direct measure of sedentary time, it was not considered in the primary analyses.

2.3.4. Total Sitting Questionnaire (T-SQ). The short-version of the International Physical Activity Questionnaire (IPAQ) was used to assess usual time sitting in total number of hours and minutes per day for both work and nonwork days [21]. The question reads, "How many hours did you spend sitting down while doing things like visiting friends, driving, reading, watching TV, or working at a desk or computer on a typical workday in the last week." In a sample of 744 adults, the test-retest reliabilities for the sitting items from this questionnaire ranged from $r=0.18$ to $r=0.95$ and criterion validity compared to the AG100 had low to moderate agreement $(r=0.07$ to $r=0.61)$ [21]. 
2.3.5. Domain-Specific Questionnaire (D-SQ). This questionnaire asks about time spent sitting in hours and minutes on a typical weekend day and weekday over the past 7 days in each of five domains: transportation, watching television, at work, using a computer at home, and leisure time not including television (e.g., visiting with friends). The testretest reliability and convergent validity compared to the AG100 for the five sitting domains range from $r=0.31$ to $r=0.91$ and $r=0.13$ to $r=0.74$, respectively. Both reliability and validity were lower for weekend days compared to weekdays [20]. To score the data, the sum of the sitting times from the five domains was used to represent daily sitting time.

2.3.6. $T V$ Viewing (TV-Q). The TV viewing question from the DS-Q was used to determine total time watching television. The question reads "please estimate how many hours per day you spend sitting while watching television."

2.4. Monitor Log and Wear Time. All participants recorded details about monitor wear in a log used to determine monitor wear time. Participants were asked to record the time they woke up in the morning, the time they put the monitors on, the time they took the monitors off, and the time they went to bed. They were also asked to indicate any times they took the monitors off during the day for greater than ten minutes. To be included in the analyses, a participant was required have at least four days of monitor wear for at least ten hours each within each period [28].

2.5. Statistical Evaluation. Twenty participants completed the study protocol. One participant was excluded from all analyses, because the participant sat in a seat where the thigh was perpendicular to the floor while seated. This resulted in erroneous standing time estimate from the AP tool. All statistical analyses were performed using R (www.rproject.org). Significance levels were set at $P<0.05$. The data were graphically examined using $\mathrm{q}-\mathrm{q}$ plots and histograms to confirm normality.

2.5.1. Effect of the Intervention: Primary Outcome Measure $A P$. To eliminate the effect of different wear times, we computed the percentage of wear time that was sedentary (i.e., (sedentary hours/total wear time) $* 100$ ) for each day. A repeated measures linear mixed model was then used to compare the differences in percent sedentary time pre- to postintervention. A separate model was also fit for percent stepping, percent standing, breaks per day (i.e., sit-tostand transitions), steps per day, and wear time. We also examined the differences pre- to postintervention for week and weekend days separately. Likelihood ratio tests were used to determine if the difference in each outcome measure preto postintervention was significant.

2.5.2. Sensitivity to Change. A repeated measures linear mixed model and likelihood ratio tests were used to analyze the differences pre- to postintervention in percent sedentary time for the AG100 and AG150. A paired $t$-test was used to examine the differences pre- to postintervention for TSQ, D-SQ, and the TV-Q. We also assessed the sensitivity and specificity for each measure compared to the AP. Based on the AP, an individual was classified as a responder (reduced sedentary time) or nonresponder (did not reduce sedentary time) during the intervention period compared to the baseline period. There was no minimum amount of change required to count as a responder. We then identified responders and nonresponders to the intervention for each of the other measures and categorized them based on the following criteria.

(1) True positives: The individual was a responder according to both the AP and the measure.

(2) True negative: The individual was a nonresponder according to both the AP and the measure.

(3) False positive: The individual was a nonresponder according to the AP but was a responder according to the measure.

(4) False negative: The individual was a responder according to the AP but was a nonresponder according to the measure.

Sensitivity was calculated as the true positives/(true positives + false negatives $) * 100$. Specificity was calculated as the true negatives/(true negatives + false positives) $* 100$. The $95 \%$ confidence intervals (CI) were calculated for both sensitivity and specificity.

2.5.3. Convergent Validity. For the third aim, we assessed the validity of the questionnaires, AG100 and AG150 for measuring baseline sedentary time per day with the AP serving as the criterion measure. Since the questionnaires ask about weekend and weekdays separately, we examined weekend and weekdays separately for the activity monitors.

We assessed bias and precision to determine validity. Bias is the average difference of the estimate from the measure (AG100, AG150, T-SQ, and D-SQ) and the AP sedentary time (minutes). A positive bias indicates the measure overestimates sedentary time, and a negative bias indicates the measure underestimates sedentary time. Precision is the inverse of variability or random error, which was examined using confidence intervals and Pearson correlations. Higher precision was indicated by higher correlations and smaller confidence intervals. For the TV-Q, we assessed the Pearson correlation between AP daily sitting and TV-Q but did not assess bias, since the TV-Q does not produce an estimate of overall sedentary time.

\section{Results}

\subsection{Efficacy of the Intervention}

3.1.1. Changes Pre- to Postintervention. Participants significantly reduced sedentary time according to the AP from $67.0 \%$ of wear time in the baseline period to $62.7 \%$ of wear time in the intervention period $(P<0.05)$ (Table 1$)$. Stepping time and steps per day significantly increased, there 
TABLE 1: ActivPAL outcome measures pre- and postintervention.

\begin{tabular}{lcccccc}
\hline & \multicolumn{2}{c}{ All days } & \multicolumn{2}{c}{ Weekday } & \multicolumn{2}{c}{ Weekend } \\
& Baseline period & Intervention period & Baseline period & Intervention period & Baseline period & Intervention period \\
\hline \% Sedentary & $67.0 \pm 13.3$ & $62.7 \pm 11.9^{*}$ & $69.4 \pm 11.1$ & $65.6 \pm 9.5^{*}$ & $61.0 \pm 16.3^{\ddagger}$ & $55.9 \pm 14.2$ \\
\% Standing & $23.2 \pm 9.7$ & $25.6 \pm 9.7$ & $21.4 \pm 8.3$ & $23.3 \pm 8.0$ & $27.5 \pm 11.6^{\ddagger}$ & $31.0 \pm 11.4$ \\
\% Stepping & $9.8 \pm 5.0$ & $11.7 \pm 4.3^{*}$ & $9.1 \pm 4.0$ & $11.1 \pm 3.7^{*}$ & $11.5 \pm 6.7^{\ddagger}$ & $13.1 \pm 5.2$ \\
Breaks per day & $53.2 \pm 21.0$ & $49.2 \pm 17.1^{*}$ & $56.2 \pm 22.4$ & $53.6 \pm 17.3$ & $46.0 \pm 15.4^{\ddagger}$ & $38.6 \pm 11.4^{*}$ \\
Steps per day & $6417 \pm 3366$ & $8167 \pm 3600^{*}$ & $6121 \pm 2495$ & $8133 \pm 3101^{*}$ & $7132 \pm 4871$ & $8247 \pm 4650$ \\
$\begin{array}{l}\text { Daily wear time } \\
\text { (hrs) }\end{array}$ & $14.1 \pm 1.9$ & $14.1 \pm 2.0$ & $14.1 \pm 1.98$ & $14.3 \pm 2.0$ & $14 \pm 1.7$ & $13.7 \pm 2.1$ \\
\hline
\end{tabular}

Note: \% Sedentary, \% Standing, and \% Stepping expressed as percent of wear time (e.g., (total sedentary time/wear time)* 100 ).

Data includes 14 participants with valid data during both the baseline and intervention period.

${ }^{\ddagger}$ Significantly different from weekdays during baseline period.

* Significantly different in intervention condition compared to baseline condition $(P<0.05)$.

TABLE 2: Monitor and questionnaire sedentary time, sensitivity, and specificity using the AP as the criterion measure.

\begin{tabular}{|c|c|c|c|c|c|}
\hline & & $\begin{array}{c}\text { Baseline period } \\
\text { mean } \pm \mathrm{SD}\end{array}$ & $\begin{array}{c}\text { Intervention period } \\
\text { mean } \pm \mathrm{SD}\end{array}$ & $\begin{array}{l}\text { Sensitivity } \\
(95 \% \mathrm{CI})\end{array}$ & $\begin{array}{l}\text { Specificity } \\
(95 \% \text { CI })\end{array}$ \\
\hline \multirow{6}{*}{ Weekday } & AP (\% Sedentary) & $68.8 \pm 8.5$ & $65.1 \pm 6.5^{*}$ & - & - \\
\hline & AG100 (\% Sedentary) & $66.4 \pm 10.2$ & $62.9 \pm 10.5$ & $80(53,100)^{\dagger}$ & $67(39,94)$ \\
\hline & AG150 (\% Sedentary) & $70.5 \pm 9.4$ & $67.1 .4 \pm 10.1$ & $70(43,97)$ & $67(39,94)$ \\
\hline & T-SQ (hours/day) & $9.3 \pm 3.3$ & $8.2 \pm 4.4$ & $80(53,100)^{\dagger}$ & $33(06,61)$ \\
\hline & D-SQ (hours/day) & $12.6 \pm 2.9$ & $11.6 \pm 2.2$ & $70(43,100)^{\dagger}$ & $33(06,61)$ \\
\hline & TV-Q (hours/day) & $2.3 \pm 1.85$ & $2.5 \pm 1.75$ & $20(0,47)$ & $100(73,100)^{\dagger}$ \\
\hline \multirow{6}{*}{ Weekend } & AP (\% Sedentary) & $60.4 \pm 15.6$ & $57.3 \pm 12.1$ & - & - \\
\hline & AG100 (\% Sedentary) & $62.7 \pm 8.9$ & $64.4 \pm 7.3$ & $67(38,95)$ & $71(43,100)^{\dagger}$ \\
\hline & AG150 (\% Sedentary) & $66.7 \pm 9.0$ & $69.0 \pm 6.2$ & $57(29,85)$ & $80(52,100)^{\dagger}$ \\
\hline & T-SQ (hours/day) & $6.2 \pm 3.1$ & $6.0 \pm 3.3$ & $57(29,85)$ & $60(32,88)$ \\
\hline & D-SQ (hours/day) & $12.1 \pm 5.0$ & $10.7 \pm 3.9$ & $57(29,85)$ & $60(32,88)$ \\
\hline & TV-Q (hours/day) & $3.4 \pm 2.14$ & $3.3 \pm 1.60$ & $43(15,71)$ & $100(72,100)^{\dagger}$ \\
\hline
\end{tabular}

Note: AP is activPAL, AG100 is ActiGraph cut-point of 100 counts $\cdot \mathrm{min}^{-1}$, AG150 is ActiGraph 150 cut-point of 150 counts $\cdot$ min $^{-1}$, T-SQ is total daily sitting questionnaire, D-SQ is domain specific questionnaire, TV-Q is the D-SQ question specifically about TV viewing.

Data included 13 participants with valid data for all measures during both the baseline and intervention period.

AP, AG100, and AG150 are expressed as a percentage (total sedentary time/wear time) to adjust for differences in wear time.

* Indicates statistically significant difference between conditions $P<0.01$.

${ }^{\dagger}$ Indicates significant sensitivity or specificity $(P<0.05)$.

was a decrease in breaks per day, and there was no significant change in standing time (Table 1). Three participants wore the AP monitor upside down on four or more days of a condition resulting in invalid data for those individuals. For two participants, the AP monitor stopped prematurely and recorded less than two days of data during one condition, leaving a total sample of $n=14$ with valid AP data both preand postintervention.

3.1.2. Differences between Weekend and Weekdays. At baseline, participants were less sedentary, stood more, had more stepping time, and took fewer breaks from sitting on weekend days compared to weekdays according to the AP $(P<0.01)$. There was no significant difference in steps per day between weekend and weekdays. On weekdays, sedentary time decreased from $69.5 \%$ of wear time in the baseline period to $65.6 \%$ of wear time in the intervention period $(P<0.05)$. This change pre- to postintervention is equivalent to a 37 -minute reduction over a 16-hour waking day. On weekend days, sedentary time was $60.9 \%$ of wear time in the baseline period, and it was $55.9 \%$ of wear time in the intervention period. This is equivalent to a 48.6 minute reduction over a 16 -hour waking day but was not a statistically significant change $(P=0.2)$.

3.2. Device and Questionnaire Sensitivity to Change. Neither AG measure (AG100 or AG150) was able to detect a statistically significant difference in sedentary time between the baseline and intervention period (Table 2). None of the questionnaires detected significant differences between the baseline and intervention period either (Table 2). To allow for a direct comparison across the measures, this analysis was done for only the individuals who had valid data for all the measures (AP, AG, and questionnaires). For this analysis, only participants with valid data from the AP, AG, and the questionnaires at both time points were included. Six 
individuals did not have valid AP data at both time points and were excluded. One AG monitor did not record a week's worth of data, leaving a total sample of 13 individuals for this analysis. However, since the power to detect change is smaller with the smaller sample size, we also examined the difference between conditions in all participants for the AG $(n=19)$ and questionnaires $(n=20)$, and the differences remained nonsignificant.

Of the 13 subjects with valid data for all measures, there were ten responders on weekdays and seven responders on weekend days according to the AP measure of sedentary time. The sensitivity, specificity, and CI's for each measure compared to the AP are shown in Table 2. The sensitivities for the AG100 and AG150 for weekdays were 80\% (CI: 50\%, $100 \%$ ) and $70 \%$ (CI: 43\%, 97\%), respectively. Specificity on weekdays was $67 \%$ (CI:39\%, 94\%) for both AG100 and AG150. Sensitivity was nominally lower (67\% and 57\%), and specificity was nominally higher (71 and 80\%) for AG100 and AG150, respectively, on weekend days compared to weekdays. Those differences were not statistically significant $(P>0.05)$.

The sensitivities and specificities for all questionnaires for weekdays and weekend days ranged from $20 \%$ to $80 \%$ and from $33 \%$ to $100 \%$, respectively. TV-Q had the lowest sensitivity but the highest specificity among the questionnaires. Both the DS-Q and T-SQ had higher sensitivity for weekdays. The sensitivity and specificity measures were lower for weekdays than weekend days for T-SQ and D-SQ, but the opposite was true for TV-Q (Table 2). Those differences were not statistically significant $(P>0.05)$.

3.3. Convergent Validity. For the monitors, we compared bias and precision overall (total week) and for weekend and weekdays separately. For the overall week, the bias (95\% CI) for the AG100 was $-3.8 \mathrm{~min},(-29$ to $22.2 \mathrm{~min}$ ). That is not significantly different from unbiased. The AG150 significantly overestimated sedentary time $31.7 \mathrm{~min}$ (7.1 to $56.3 \mathrm{~min})$.

3.3.1. AG Weekend and Weekday. For weekdays, the AG100 significantly underestimated sedentary time by $40 \mathrm{~min}$ ( -69.7 to $-8.3 \mathrm{~min}$ ), and there was no significant difference between the AP and AG150 with an average difference of $1.4 \mathrm{~min},(-29$ to $31.9 \mathrm{~min})$. The correlation on weekdays between the AP and AG100 was $(r=0.52)(P<0.05)$, and between the AP and AG150, it was $(r=0.55)(P<0.05)$.

For weekend days, the bias was $20.8 \mathrm{~min}$ ( -32 to $74 \mathrm{~min}$ ) for the AG100. The AG150 significantly overestimated sedentary time with a bias of $58.3 \mathrm{~min}$ (6.7 to $93.1 \mathrm{~min}$ ) on weekend days. AP estimates of sitting were correlated with the AG150 $(r=0.68)$ and the AG100 $(r=0.68)$ for weekend days $(P<0.05)$.

3.3.2. Questionnaires. The T-SQ underestimated sitting time, but it was not significantly different than the AP for weekdays, with an average difference of $40.5 \mathrm{~min}(-125.2$ to 22.3). The correlation was not statistically significantly different from zero $(r=0.41)$. The estimate of sitting time from the T-SQ was $147.4 \mathrm{~min}(-228.3$ to -66.6$)$ less than the AP for weekend days $(P<0.05)$. The correlation between sitting time from the T-SQ and AP was significant for weekend days $(r=0.55)(P<0.05)$.

The D-SQ significantly overestimated sitting time for both weekend and weekdays. On weekdays, the D-SQ overestimated sitting time by $176 \mathrm{~min}$ ( 96.1 to $256.9 \mathrm{~min}$ ). Similarly, on weekend days, sitting time was overestimated by $157.6 \mathrm{~min}$ (22.1 to $293.0 \mathrm{~min}$ ). The correlation between the AP and D-SQ was not significant for either or weekdays $(r=0.30)$ or weekend days $(r=0.17)$. The correlation between the AP and TV-Q was not significant for either weekdays $(r=0.07)$ or weekend days $(r=-0.11)$.

\section{Discussion}

This study addressed two important knowledge gaps in the field of sedentary behavior and health. First, it provides empirical evidence that it is possible to reduce free-living sedentary behavior among overweight and obese, nonexercising adults. Participants decreased sedentary time by $\sim 5 \%$, which is equivalent to 48 minutes over a 16-hour waking day. Second, this study identified a measurement tool that is sensitive to change in sedentary behavior and provided a comparison of two commonly used accelerometer-based monitors and two self-report questionnaires.

4.1. Feasibility of Sedentary Behavior Intervention. To date, only two published intervention trials targeting sedentary time reductions are available in adults $[29,30]$. Our results are similar to these trials despite differences in the study sample demographics, intervention targets, and measurement tools. Otten et al. targeted TV viewing among overweight and obese individuals who watch TV $>3$ hours per day and showed a 3.8\% decrease in sedentary time [29]. Their study targeted only one sedentary domain (TV viewing) and the primary outcome was percent of time in sedentary activities according to the Sensewear arm band [29]. Gardiner and colleagues [30] completed a similar study to the current one. They included older adults who completed a 7-day baseline period followed by a 7-day intervention targeting sedentary time. They reported a $3.2 \%$ decrease in sedentary time [30]. They did not exclude participants who were participating in MVPA at baseline, and occupational sitting was not a target for their intervention, since many participants were retired. The primary outcome measure was the AG100 estimate of sedentary time [30]. To our knowledge, our study is the first to show a significant reduction of freeliving sedentary time using a targeted intervention among nonexercising office workers and the first to use the AP monitor as an objective tool to assess sedentary time in an intervention study. Participants replaced sedentary time by increasing stepping $(P<0.01)$ and standing time $(P=0.06)$. Breaks from sedentary time significantly decreased in the intervention period, which is of concern given the evidence that more breaks from sitting may be beneficial for metabolic health [22]. However, since sedentary time was replaced with standing, there will naturally be less opportunity for sitto-stand transitions. Thus, in future research, both breaks 
TABLE 3: Individual responsiveness to intervention for each measure.

\begin{tabular}{|c|c|c|c|c|c|c|c|c|c|c|c|c|}
\hline \multirow[b]{2}{*}{$\begin{array}{l}\vec{\Xi} \\
\vec{\Xi} \\
: \\
: \\
\Xi \\
\Xi\end{array}$} & \multicolumn{5}{|c|}{ Week days } & \multirow[b]{2}{*}{$\begin{array}{l}\text { O } \\
\stackrel{1}{2}\end{array}$} & \multicolumn{6}{|c|}{ Weekend days } \\
\hline & \& & 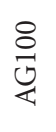 & 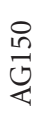 & $\begin{array}{l}O \\
\text { D } \\
H\end{array}$ & $\begin{array}{l}0 \\
0 \\
1 \\
1\end{array}$ & & \& & 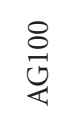 & $\begin{array}{l}\text { in } \\
\text { 足 } \\
\end{array}$ & $\begin{array}{l}O \\
\text { 足 } \\
H\end{array}$ & $\begin{array}{l}0 \\
0 \\
1 \\
0\end{array}$ & $\begin{array}{l}O \\
\lambda^{1} \\
\lambda^{\prime}\end{array}$ \\
\hline 1 & + & + & + & + & + & - & + & + & + & + & - & - \\
\hline 2 & + & + & + & + & + & - & + & + & + & - & + & + \\
\hline 3 & + & + & + & + & + & - & - & - & - & - & - & - \\
\hline 4 & + & + & + & + & - & - & - & - & - & - & - & - \\
\hline 5 & - & - & - & - & - & - & + & - & - & + & + & + \\
\hline 6 & + & + & - & + & + & - & + & + & + & + & + & + \\
\hline 7 & + & + & + & + & - & - & *NA & *NA & ${ }^{*} \mathrm{NA}$ & ${ }^{*} \mathrm{NA}$ & ${ }^{*} \mathrm{NA}$ & *NA \\
\hline 8 & + & - & + & + & + & - & - & - & - & - & - & - \\
\hline 9 & - & - & - & - & - & - & + & - & - & - & - & - \\
\hline 10 & + & - & - & - & - & + & + & + & + & - & - & - \\
\hline 11 & - & - & - & - & - & - & + & + & + & + & + & - \\
\hline 12 & + & + & + & - & + & + & - & - & - & - & - & - \\
\hline 13 & + & - & - & + & + & - & - & - & - & - & - & - \\
\hline
\end{tabular}

Note: + represents responder (reduced sedentary time pre- to postintervention) and - represents nonresponders (did not reduce sedentary time pre- to postintervention) for each individual.

AP refers to activPAL monitor, AG100 refers to the ActiGraph cut-point of 100 counts $\cdot$ min $^{-1}$, AG150 refers to the ActiGraph cut-point of 150 counts $\cdot$ min $^{-1}$, $\mathrm{T}-\mathrm{SQ}$ is a single-item total sedentary time questionnaire and D-SQ is a 5-domain sedentary time questionnaire. TV-Q refers to D-SQ question about TVviewing only.

$* \mathrm{NA}=$ no valid AP data for weekend.

from sitting and changes in absolute sedentary time must be used as outcome measures in evaluation of effectiveness of interventions designed to reduce sedentary time.

4.2. Intervention Strategies. At the end of the study, participants were asked to report which strategies were most effective for reducing sedentary time. All participants (19/19) reported that the pedometer was helpful, but participants who averaged $<5000$ steps per day at baseline found the 7500 goal to be too high. Future research should consider setting more modest incremental step goals based on the participant's baseline level of steps. While the intervention targeted sedentary time, participants reported that the step goal was helpful, because it provided instant quantitative selfmonitoring feedback. Based on these findings, a device that tracks and provides instant quantitative feedback specific to sedentary time may help participants reduce sedentary time. Approximately half (10/19) of the participants found the hourly checklist (where they reported whether they had stood for five or more minutes each hour) to be helpful, and they reported completing it as they finished activities. The remaining nine only completed the hourly checklist either daily or every few days. These simple strategies, targeting small behavioral changes and providing self-monitoring tools, may be useful for future interventions targeting reductions in sedentary time.

4.3. Sensitivity of Measurement Tools. The AP was used as the criterion to differentiate responders to the intervention from nonresponders [23]. In this study, we confirmed the
AP was sensitive to the reductions in sedentary time, but the AG and the self-report questionnaires were not. A novel aspect of this study was that it examined the sensitivity and specificity of the various measures for detecting changes in behavior. In intervention studies, it is important to use measures with high sensitivity and specificity to insure that changes can be detected and to minimize sample size requirements. Sensitivity reflects the ability of a measure to correctly classify true behavior change. For example, the sensitivity of the AG100 was $67 \%$ for weekend days. That is, one-third of subjects who actually changed their behavior according to the AP were not classified as changing their behavior according to the AG100. The specificity was lowest for the D-SQ and T-SQ, indicating that participants were more likely to report they changed behavior when they were actually nonresponders to the intervention (according to the AP). In addition, the misclassifications across measures were not occurring for the same individuals. For example, five individuals were misclassified according to the D-SQ, T-SQ, and AG150 for weekend days, but it was not the same five individuals for each measure (see Table 3).

The results comparing sensitivity to change of the AG and $\mathrm{AP}$ are consistent with our previous results which used 6 hours of direct observation as the criterion measure [23]. Gardiner and colleagues previously reported the AG was modestly sensitive to change and detected a significant decrease in sedentary time (3.2\%) using the AG100 [31]. Their study included 48 individuals, which suggests that the AG may be able to detect change in a larger sample. However, in the current study, eight minutes more sitting 


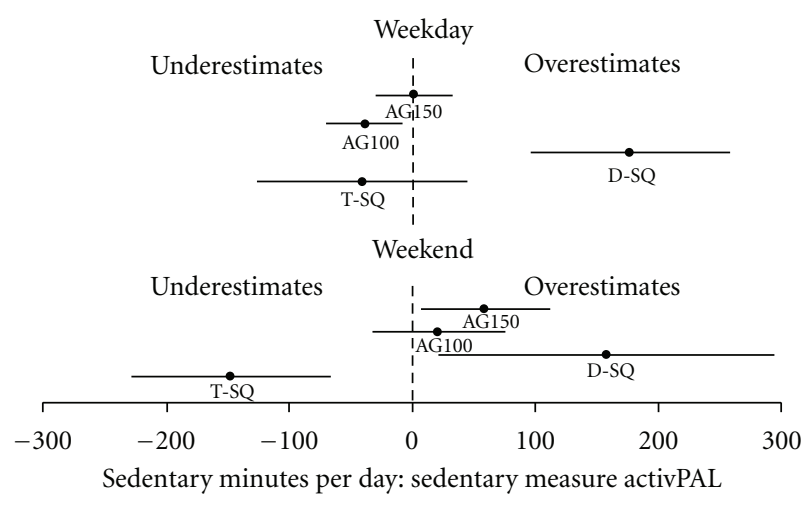

FIGURE 1: Illustration of the under- and overestimation of sedentary time for each measure compared to the activPAL monitor for (a) weekend (b) weekdays. The closed circles are the bias and the lines illustrate the $95 \%$ confidence intervals. AG100 refers to the ActiGraph cut-point of 100 counts $\cdot \mathrm{min}^{-1}$, AG150 refers to the ActiGraph cut-point of 150 counts $\cdot \mathrm{min}^{-1}$, T-SQ is a singleitem total sedentary time questionnaire, and D-SQ is a 5-domain sedentary time questionnaire. Data includes 13 participants with valid data for all measures during both the baseline and intervention period.

time on weekend days was recorded with the AG measures in the intervention period compared to the baseline period. In contrast, the AP recorded 54 minutes less sitting on weekend days in the intervention period. Participants spent more time standing on weekends ( $31 \%$ of AP wear time) than on weekdays $(23.4 \%$ of wear time) in the intervention period. This suggests that the AG does not distinguish standing from sitting. This is not surprising, since the AG device is not designed to differentiate postures. If a person is standing still or standing with small amounts of movement, this will be interpreted as sedentary time using the AG cutpoint method. This will cause measurement problems for interventions, where participants are encouraged to replace sitting with standing.

4.4. Convergent Validity of AG. The AG100 cut-point was more accurate than the AG150, and that differs from our previous work which used the same subjects and direct observation as the criterion measure (Figure 1). In the current study, there were differences in the accuracy of the cut-points depending on how much the participant was sedentary. When sedentary time was highest (on weekdays $\sim 67 \%$ ), the AG150 was not different from the AP, while the AG100 significantly underestimated sedentary time. When sedentary time was lower (on weekend days $\sim 62.7 \%$ ), the AG150 significantly overestimated sedentary time, while the AG100 was not significantly different from the AP. In our previous study, participants were directly observed while at work over a 6-hour period, and the percent of time sedentary according to the AP was considerably higher for both the baseline period $(79.5 \%)$ and intervention period $(66.5 \%)$ than the current study, which may explain the discrepancy. Additionally, it should be noted that following an intervention designed to increase standing and decrease sedentary time, the AG150 may misclassify standing as sedentary behavior and inflate sedentary time. In a highly sedentary population, the AG100 may underestimate sedentary time.

Accuracy using the AG100 and AG150 was slightly different, and both were equally precise with the 95\% confidence intervals of about 50 minutes. While the accuracy of a given cut-point may change depending on the level of sedentary behavior, the precision will not. Lower precision increases sample size requirements for intervention trials. It is also important to note that the AG monitors did detect significant differences pre- to postintervention for minutes in MVPA, which increased significantly during the intervention period from $16.1 \mathrm{~min}$ to $24.6 \mathrm{~min}(P<0.01)$. To date, limited work has been done validating MVPA estimates from the AP. Therefore, intervention studies targeting both sedentary time and MVPA should consider using the AG.

4.5. Convergent Validity of Questionnaires. To our knowledge, this is the first study comparing questionnaire estimates of sedentary time to the AP. In contrast to the T-SQ, which underestimated sedentary time, the D-SQ overestimated sedentary time. Therefore, it is very important to consider the type of questionnaire when attempting to compare prevalence estimates across populations. Clemes et al. compared two sedentary behavior questionnaires to the AG100 [32]. Similar to our results, they reported the single-item T-SQ underestimated sedentary time by over two hours on weekend days. In the current study, the difference between the T-SQ and the AP was not significant on weekdays, while Clemes et al. did report a significant underestimation of sedentary time [32]. They reported no significant difference for the D-SQ compared to the AG100, which is different than what we reported when comparing the questionnaires to the AP. While participants were instructed to avoid doublereporting of time in multiple domains, it is possible that this occurred. Another explanation is that participants were awake for more time than they wore the monitors, which leaves potential time for participants to be sedentary that is not captured by the monitors. In the Clemes et al. [32] study, participants reported in a diary how much they sat each day during the week, which may have improved their awareness of sedentary time. Further, while the average difference in their study was small, they reported very wide limits of agreement using a Bland-Altman analyses (weekday $=-382.0$ to $354.6 \mathrm{~min}$; weekend day $=-578.5$ to $570.2 \mathrm{~min}$ ) which is consistent with the large individual differences in the present study. Only considering one domain (TV viewing) was not sufficient to detect change in behavior and was not correlated with overall sedentary time. While the evidence linking high levels of TV viewing to poor health outcomes is robust, a more comprehensive measure of sedentary time should be used by future studies that examine the doseresponse relationships of overall sedentary time and health.

This study has important limitations that should be noted. We used a ten hour cutoff to define a valid day using the activity monitors. This is considered best practice for accelerometer studies and previous validation studies of sedentary questionnaires [20], but the 10-hour criterion was originally designed for studies that primarily measure 
MVPA [28]. Future work should examine if this is a valid criterion for determining minimum wear time needed in sedentary behavior studies. Future research, using a larger sample size, should examine the difference in estimates of sedentary time using different daily wear time criteria. The second limitation is that the sample was small and homogenous, but it is worth pointing out that subjects in this study are probably similar to those who will be targeted for future intervention (overweight/obese, nonexercising, and sedentary occupations). It is important to note that the results can only be generalized to a similar population of highly sedentary, overweight, and nonexercising office workers. Finally, this study demonstrates that short-term, free-living sedentary time reductions are possible. However, while the change we observed was statistically significant, a $\sim 5 \%$ (48 minute) reduction in sedentary time per day may not be sufficient to elicit health benefits even if sustained for a longer duration. Future research is needed to explore the health benefits of longer-term reductions in sedentary time.

The strengths of this study are the within subject design that allowed us to explore key measurement limitations in the literature in unique ways. Particularly, the sensitivity to change analyses using sensitivity and specificity will inform researchers of sample size requirements for future intervention trials. In the current study, we used the AP as a criterion for changes in behavior and for measuring sedentary time. The AP has been shown to correctly classify free-living sedentary time over $97.2 \%$ of the time $[23,24]$. While this is not $100 \%$ accurate, we believe the effects on the comparisons across measures are small though they may exist. To date, few studies have used the AP monitor, or a comparably accurate criterion measure, to assess the efficacy of interventions or to examine the convergent validity of sedentary time measures. In addition, our study is the first known sedentary behavior intervention study in adults to use the AP as the primary outcome measure. Finally, we provide a number of strategies and behavior change tools for future interventions that target reductions in sedentary time.

In conclusion, this study confirmed that the AP monitor is sensitive to change, and the $A G$ monitor and selfreport questionnaires are less sensitive. We provide data that improves our understanding of the measurement properties of devices and self-report tools. These data will help inform sample size estimates for future interventions. The AG100 was more accurate when sedentary time was lower, while the AG150 was more accurate when sedentary time was higher. This discrepancy highlights the inherent limitations of estimating sedentary time using a simple cut-point from a waist-mounted accelerometer. When possible, researchers should use a device that is specifically designed to measure posture for intervention studies that target sedentary time. In addition, we showed that a $\sim 50$ minute per day reduction in sedentary time is possible using targeted messages to replace sedentary time with standing and light-intensity activity. While there is evidence linking sedentary behavior to health, there remains a paucity of controlled trials examining the effect of reducing sitting time on health outcomes [25]. In the future, long-term randomized controlled trial studies are necessary to demonstrate the effect of reducing sedentary time on the cardiometabolic risk factors associated with chronic diseases.

\section{Disclosure}

P. Freedson is a paid member of the Actigraph Scientific Advisory Board. No other author has disclosures to report.

\section{Acknowledgment}

Funding was provided by an American College of Sports Medicine Foundation Doctoral Student Grant awarded to S. Kozey-Keadle.

\section{References}

[1] N. Owen, G. N. Healy, C. E. Matthews, and D. W. Dunstan, "Too much sitting: the population health science of sedentary behavior," Exercise and Sport Sciences Reviews, vol. 38, no. 3, pp. 105-113, 2010.

[2] C. Tudor-Locke, W. D. Johnson, and P. T. Katzmarzyk, "Frequently reported activities by intensity for U.S. adults: the American time use survey," American Journal of Preventive Medicine, vol. 39, no. 4, pp. e13-e20, 2010.

[3] C. E. Matthews, K. Y. Chen, P. S. Freedson et al., "Amount of time spent in sedentary behaviors in the United States, 20032004," American Journal of Epidemiology, vol. 167, no. 7, pp. 875-881, 2008.

[4] C. E. Matthews, B. E. Ainsworth, C. Hanby et al., "Development and testing of a short physical activity recall questionnaire," Medicine and Science in Sports and Exercise, vol. 37, no. 6, pp. 986-994, 2005.

[5] G. N. Healy, D. W. Dunstan, J. Salmon et al., "Objectively measured light-intensity physical activity is independently associated with 2-h plasma glucose," Diabetes Care, vol. 30, no. 6, pp. 1384-1389, 2007.

[6] G. N. Healy, C. E. Matthews, D. W. Dunstan, E. A. Winkler, and N. Owen, "Sedentary time and cardio-metabolic biomarkers in US adults: NHANES 2003-06," European Heart Journal, vol. 32, no. 5, pp. 590-597, 2011.

[7] B. M. Lynch, D. W. Dunstan, G. N. Healy, E. Winkler, E. Eakin, and N. Owen, "Objectively measured physical activity and sedentary time of breast cancer survivors, and associations with adiposity: findings from NHANES (2003-2006)," Cancer Causes and Control, vol. 21, no. 2, pp. 283-288, 2010.

[8] A. A. Thorp, N. Owen, M. Neuhaus, and D. W. Dunstan, "Sedentary behaviors and subsequent health outcomes in adults: a systematic review of longitudinal studies, 19962011," American Journal of Preventive Medicine, vol. 41, no. 2, pp. 207-215, 2011.

[9] F. B. Hu, "Sedentary lifestyle and risk of obesity and type 2 diabetes," Lipids, vol. 38, no. 2, pp. 103-108, 2003.

[10] F. B. Hu, M. F. Leitzmann, M. J. Stampfer, G. A. Colditz, W. C. Willett, and E. B. Rimm, "Physical activity and television watching in relation to risk for type 2 diabetes mellitus in men," Archives of Internal Medicine, vol. 161, no. 12, pp. 15421548, 2001.

[11] H. J. F. Helmerhorst, K. Wijndaele, S. Brage, N. J. Wareham, and U. Ekelund, "Objectively measured sedentary time may predict insulin resistance independent of moderate- and vigorous-intensity physical activity," Diabetes, vol. 58, no. 8, pp. 1776-1779, 2009. 
[12] D. W. Dunstan, E. L. M. Barr, G. N. Healy et al., "Television viewing time and mortality: the australian diabetes, obesity and lifestyle study (ausdiab)," Circulation, vol. 121, no. 3, pp. 384-391, 2010.

[13] U. Ekelund, S. Brage, H. Besson, S. Sharp, and N. J. Wareham, "Time spent being sedentary and weight gain in healthy adults: reverse or bidirectional causality?" American Journal of Clinical Nutrition, vol. 88, no. 3, pp. 612-617, 2008.

[14] U. Ekelund, S. Brage, S. J. Griffin, and N. J. Wareham, “Objectively measured moderate- and vigorous-intensity physical activity but not sedentary time predicts insulin resistance in high-risk individuals," Diabetes Care, vol. 32, no. 6, pp. 10811086, 2009.

[15] F. B. Hu, T. Y. Li, G. A. Colditz, W. C. Willett, and J. E. Manson, "Television watching and other sedentary behaviors in relation to risk of obesity and type 2 diabetes mellitus in women," Journal of the American Medical Association, vol. 289, no. 14, pp. 1785-1791, 2003.

[16] A. Grøntved and F. B. Hu, “Television viewing and risk of type 2 diabetes, cardiovascular disease, and all-cause mortality: a meta-analysis," Journal of the American Medical Association, vol. 305, no. 23, pp. 2448-2455, 2011.

[17] E. M. Blass, D. R. Anderson, H. L. Kirkorian, T. A. Pempek, I. Price, and M. F. Koleini, "On the road to obesity: television viewing increases intake of high-density foods," Physiology and Behavior, vol. 88, no. 4-5, pp. 597-604, 2006.

[18] B. K. Clark, T. Sugiyama, G. N. Healy et al., "Sociodemographic correlates of prolonged television viewing time in australian men and women: the AusDiab study," Journal of Physical Activity and Health, vol. 7, no. 5, pp. 595-601, 2010.

[19] B. K. Clark, G. N. Healy, E. A. H. Winkler et al., "Relationship of television time with accelerometer-derived sedentary time: NHANES," Medicine and Science in Sports and Exercise, vol. 43, pp. 822-828, 2011.

[20] A. L. Marshall, Y. D. Miller, N. W. Burton, and W. J. Brown, "Measuring total and domain-specific sitting: a study of reliability and validity," Medicine and Science in Sports and Exercise, vol. 42, no. 6, pp. 1094-1102, 2010.

[21] C. L. Craig, A. L. Marshall, M. Sjöström et al., "International physical activity questionnaire: 12-Country reliability and validity," Medicine and Science in Sports and Exercise, vol. 35, no. 8, pp. 1381-1395, 2003.

[22] G. N. Healy, B. K. Clark, E. A. Winkler, P. A. Gardiner, W. J. Brown, and C. E. Matthews, "Measurement of adults' sedentary time in population-based studies," American Journal of Preventive Medicine, vol. 41, no. 2, pp. 216-227, 2011.

[23] S. Kozey-Keadle, A. Libertine, K. Lyden, J. Staudenmayer, and P. S. Freedson, "Validation of wearable monitors for assessing sedentary behavior," Medicine and Science in Sports and Exercise, vol. 43, no. 8, pp. 1561-1567, 2011.

[24] P. M. Grant, C. G. Ryan, W. W. Tigbe, and M. H. Granat, "The validation of a novel activity monitor in the measurement of posture and motion during everyday activities," British Journal of Sports Medicine, vol. 40, no. 12, pp. 992-997, 2006.

[25] N. Owen, T. Sugiyama, E. E. Eakin, P. A. Gardiner, M. S. Tremblay, and J. F. Sallis, "Adults' sedentary behavior: determinants and interventions," American Journal of Preventive Medicine, vol. 41, no. 2, pp. 189-196, 2011.

[26] C. Tudor-Locke and D. R. Bassett, "How many steps/day are enough? Preliminary pedometer indices for public health," Sports Medicine, vol. 34, no. 1, pp. 1-8, 2004.

[27] P. S. Freedson, E. Melanson, and J. Sirard, "Calibration of the computer science and applications, Inc. accelerometer,"
Medicine and Science in Sports and Exercise, vol. 30, no. 5, pp. 777-781, 1998.

[28] R. P. Troiano, D. Berrigan, K. W. Dodd, L. C. Mâsse, T. Tilert, and M. Mcdowell, "Physical activity in the United States measured by accelerometer," Medicine and Science in Sports and Exercise, vol. 40, no. 1, pp. 181-188, 2008.

[29] J. J. Otten, K. E. Jones, B. Littenberg, and J. Harvey-Berino, "Effects of television viewing reduction on energy intake and expenditure in overweight and obese adults: a randomized controlled trial," Archives of Internal Medicine, vol. 169, no. 22, pp. 2109-2115, 2009.

[30] P. A. Gardiner, E. G. Eakin, G. N. Healy, and N. Owen, "Feasibility of reducing older adults' sedentary time," American Journal of Preventive Medicine, vol. 41, no. 2, pp. 174-177, 2011.

[31] P. A. Gardiner, B. K. Clark, G. N. Healy et al., "Measuring older adults' sedentary time: reliability, validity and responsiveness," Medicine and Science in Sports and Exercise, vol. 43, no. 11, pp. 2127-2133, 2011.

[32] S. A. Clemes, B. David, Y. Zhao, X. Han, and W. Brown, "Validity of two self-report measures of sitting time," Journal of Physical Activity and Health. In press. 


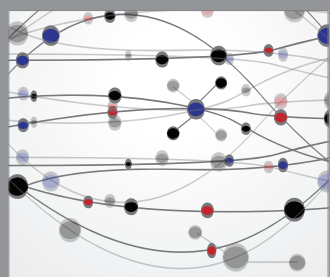

The Scientific World Journal
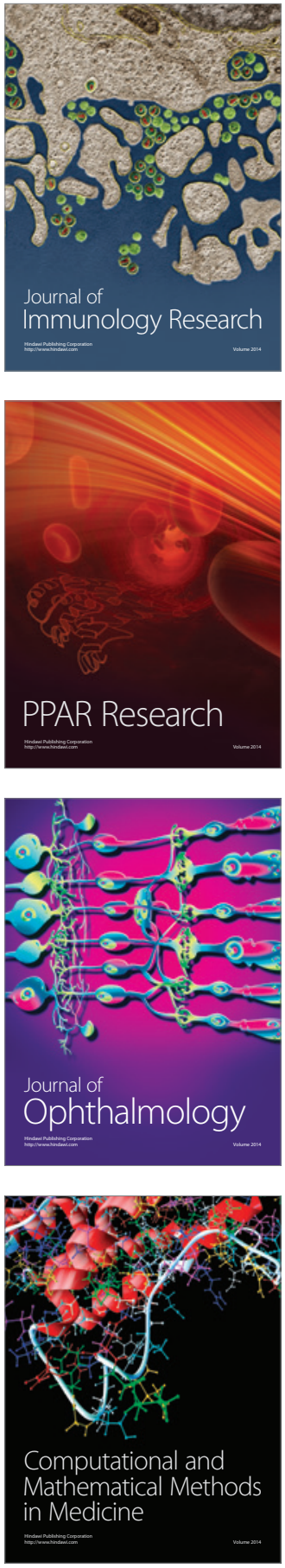

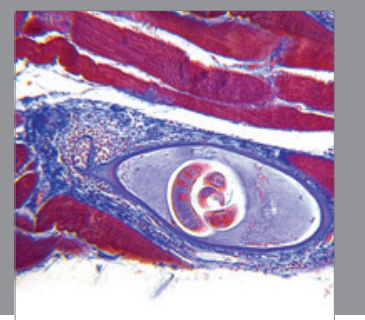

Gastroenterology

Research and Practice
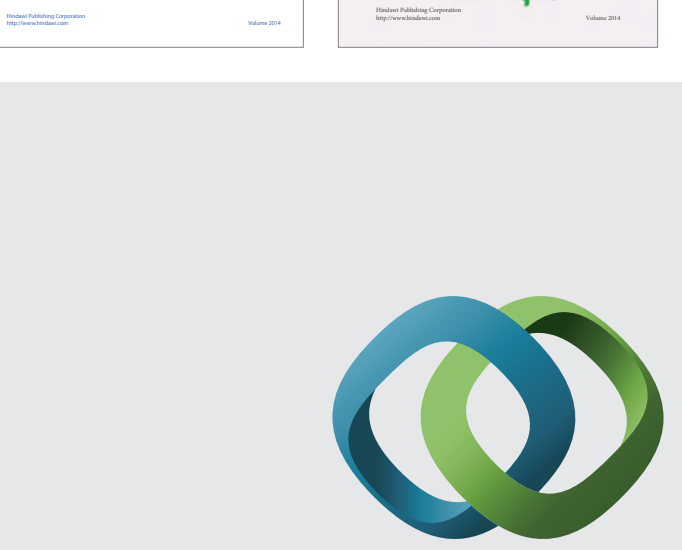

\section{Hindawi}

Submit your manuscripts at

http://www.hindawi.com
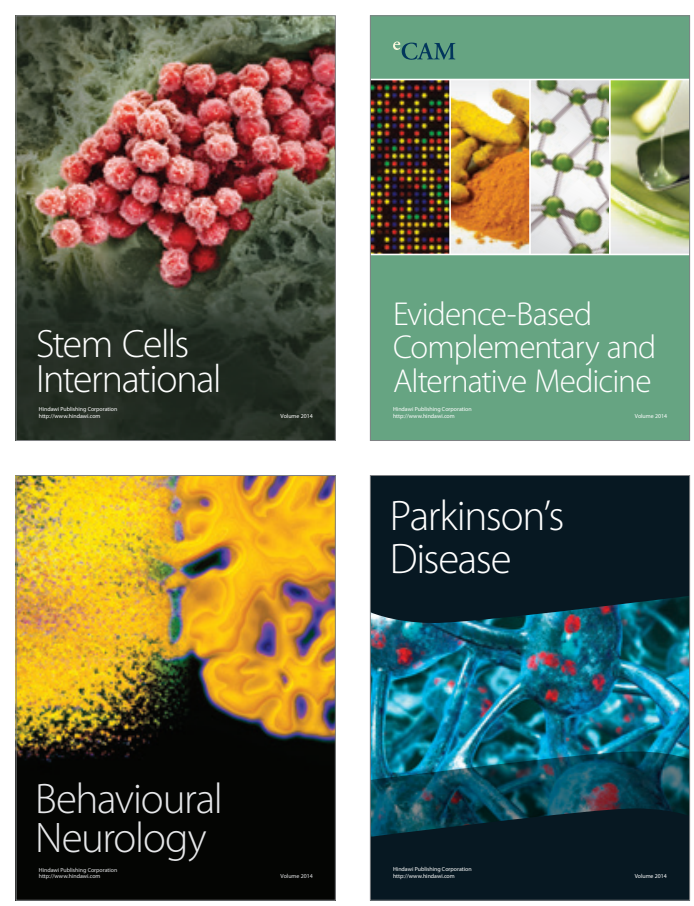

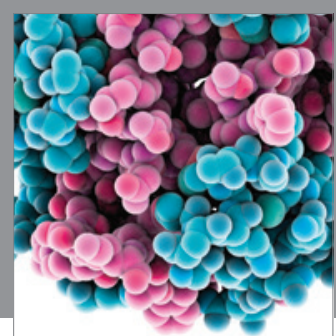

Journal of
Diabetes Research

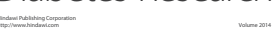

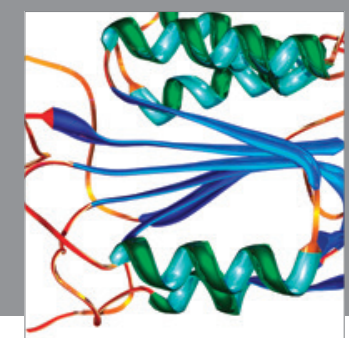

Disease Markers
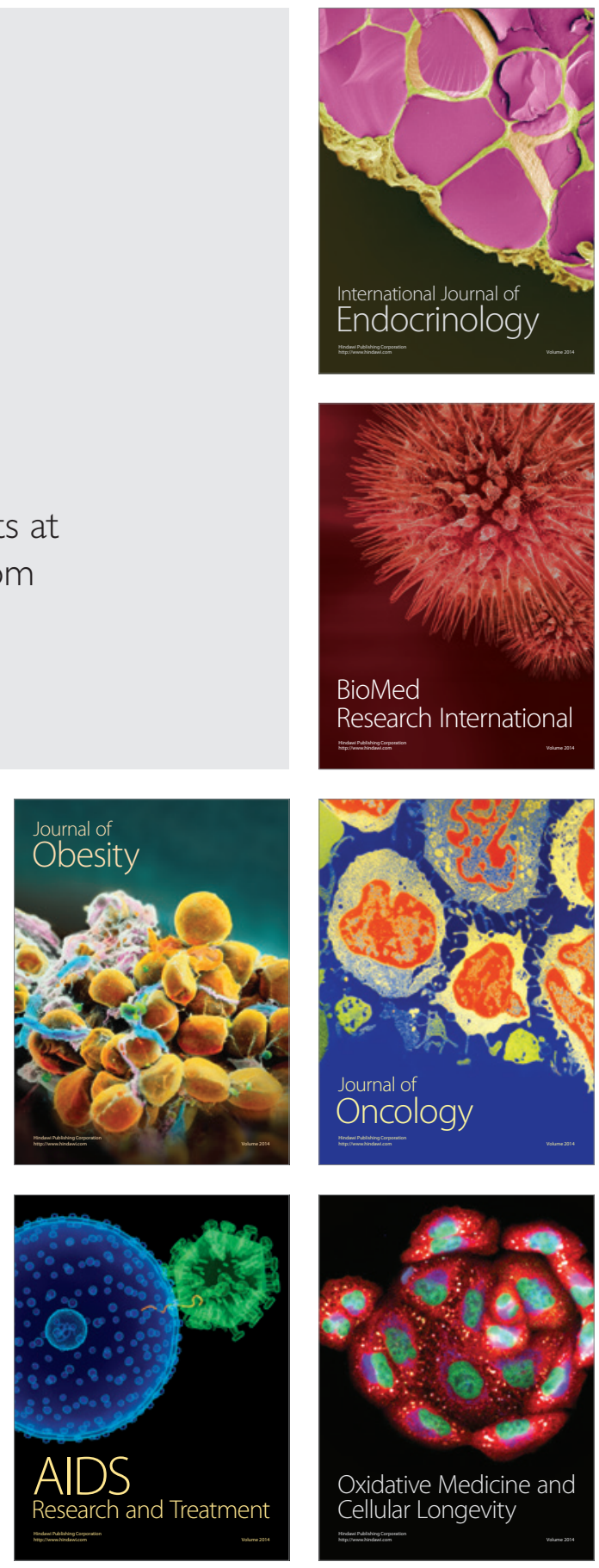